\title{
Inverse Scattering Problem for the Maxwell's Equations
}

\author{
A. G. Ramm* \\ Mathematics Department, Kansas State University, \\ Manhattan, KS 66506-2602, USA
}

\begin{abstract}
Inverse scattering problem is discussed for the Maxwell's equations. A reduction of the Maxwell's system to a new Fredholm second-kind integral equation with a scalar weakly singular kernel is given for electromagnetic (EM) wave scattering. This equation allows one to derive a formula for the scattering amplitude in which only a scalar function is present. If this function is small (an assumption that validates a Born-type approximation), then formulas for the solution to the inverse problem are obtained from the scattering data: the complex permittivity $\epsilon^{\prime}(x)$ in a bounded region $D \subset \mathbb{R}^{3}$ is found from the scattering amplitude $A(\beta, \alpha, k)$ known for a fixed $k=\omega \sqrt{\epsilon_{0} \mu_{0}}>0$ and all $\beta, \alpha \in S^{2}$, where $S^{2}$ is the unit sphere in $\mathbb{R}^{3}, \epsilon_{0}$ and $\mu_{0}$ are constant permittivity and magnetic permeability in the exterior region $D^{\prime}=\mathbb{R}^{3} \backslash D$. The novel points in this paper include: i) A reduction of the inverse problem for vector EM waves to a vector integral equation with scalar kernel without any symmetry assumptions on the scatterer, ii) A derivation of the scalar integral equation of the first kind for solving the inverse scattering problem, and iii) Presenting formulas for solving this scalar integral equation. The problem of solving this integral equation is an ill-posed one. A method for a stable solution of this problem is given.
\end{abstract}

Keywords and phrases: Electromagnetic waves, Maxwell's equations, wave scattering, inverse scattering

Mathematics Subject Classification: 35J10, 70F10, 74J25, 81U40, 81V05

\section{Introduction}

There is a large literature on electromagnetic wave (EM) scattering ([1]- [3]), to name a few books. In [4] (see also [5]-[10] and references therein), wave scattering theory is developed for small bodies of arbitrary shapes. The idea of this paper is to reduce the Maxwell's system to one Fredholm-type integral equations for the vector $E$ of eletric field and use this equation for solving inverse scattering problem. Let $\epsilon^{\prime}(x)=\epsilon(x)+\frac{i \sigma(x)}{\omega}$, where $\sigma(x)$ is the conductivity and $\epsilon(x)$ is the permittivity. It is assumed that $\epsilon(x)=\epsilon_{0}$ in $D^{\prime}=\stackrel{\omega}{\mathbb{R}^{3}} \backslash D$, i.e., outside of an arbitrary large but finite domain $D, \sigma(x)=0$ outside $D$, and the magnetic permeability $\mu=\mu_{0}$ in $\mathbb{R}^{3}$, where $\epsilon_{0}$ and $\mu_{0}$ are constants. We assume that $\epsilon^{\prime}(x)$ is twice continuously differentiable function. The inverse scattering problem consists in finding

${ }^{*}$ Corresponding author. E-mail: ramm@math.ksu.edu 
complex permittivity $\epsilon^{\prime}(x)=\epsilon(x)+\frac{i \sigma(x)}{\omega}$ from the scattering amplitude $A(\beta, \alpha, k)$, known at a fixed wavenumber $k>0$ and all directions $\alpha$ of the incident plane wave and all directions $\beta$ of the scattered waves. In Section 2 this integral equation (2.15) is derived. In Section 3 equation (2.15) is used for a study of inverse scattering problem. The original ideas in this paper include equation (2.15) and inversion procedure in Section 3, see equations (3.8)-(3.13). The novel points in this paper include: i) A reduction of the inverse problem for vector EM waves to a vector integral equation with a scalar kernel without any symmetry assumptions on the scatterer, ii) A derivation of a scalar integral equation for solving the inverse scattering problem, and in presenting formulas for solving this integral equation. The problem of solving this integral equation is an ill-posed one. A method for a stable solution of this problem is given. The results are formulated in Lemmas 2.1 and 2.2 in Section 2 and Theorems 1,2 in Section 3.

\section{EM wave scattering by a body}

Let $D$ be a finite body filled with a material that has complex permittivity $\epsilon^{\prime}(x)$ and constant magnetic permeability $\mu_{0}$.

The Maxwell's equations are:

$$
\nabla \times E=i \omega \mu H, \quad \nabla \times H=-i \omega \epsilon^{\prime}(x) E \quad \text { in } \mathbb{R}^{3},
$$

where $\omega>0$ is the frequency, $\mu=\mu_{0}$ is the constant in $\mathbb{R}^{3}$ magnetic permeability, $\epsilon^{\prime}(x)=\epsilon_{0}>0$ in $D^{\prime}$, $\epsilon_{0}$ is a constant,

$$
\epsilon^{\prime}(x)=\epsilon(x)+i \frac{\sigma(x)}{\omega}, \quad \sigma(x) \geq 0,
$$

$\epsilon^{\prime}(x) \neq 0 \forall x \in \mathbb{R}^{3}, \epsilon^{\prime}(x) \in C^{2}\left(\mathbb{R}^{3}\right)$ is a twice continuously differentiable function, $\epsilon(x)=\epsilon_{0}$ in $D^{\prime}$, and the conductivity $\sigma(x)=0$ in $D^{\prime}$. From $(2.1)$ one gets

$$
\begin{gathered}
\nabla \times \nabla \times E=K^{2}(x) E, \quad H=\frac{\nabla \times E}{i \omega \mu}, \\
K^{2}(x):=\omega^{2} \epsilon^{\prime}(x) \mu .
\end{gathered}
$$

We seek the solution of the equation

$$
\nabla \times \nabla \times E=K^{2}(x) E
$$

satisfying the radiation condition:

$$
E(x)=E_{0}(x)+v,
$$

where $E_{0}(x)$ is the plane wave,

$$
E_{0}(x)=\mathcal{E} e^{i k \alpha \cdot x}, \quad k=\frac{\omega}{c},
$$

$c=\frac{1}{\sqrt{\epsilon \mu}}$ is the wave velocity in the homogeneous medium outside $D, \epsilon=\epsilon_{0}=$ const is the dielectric parameter in the outside region $D^{\prime}, \mu=\mu_{0}=$ const in $\mathbb{R}^{3}, \alpha \in S^{2}$ is the incident direction of the plane wave, $\mathcal{E} \cdot \alpha=0, \mathcal{E}$ is a constant vector, and the scattered field $v$ satisfies the radiation condition

$$
\frac{\partial v}{\partial r}-i k v=o\left(\frac{1}{r}\right), \quad r=|x| \rightarrow \infty
$$

uniformly in directions $\beta:=\frac{x}{r}$. If $E$ is found, then the pair $\{E, H\}$, where

$$
H=\frac{\nabla \times E}{i \omega \mu},
$$


solves our scattering problem. Our goal is to derive a Fredholm second-kind integral equation for $E$. We follow closely in this derivation the presentation in [8]. Let us rewrite equation (2.4) as

$$
-\triangle E+\nabla(\nabla \cdot E)-k^{2} E-p(x) E=0,
$$

where

$$
p(x):=K^{2}(x)-k^{2}, \quad p(x)=0 \text { in } D^{\prime} .
$$

Note that

$$
\operatorname{Im} K^{2}(x)=\operatorname{Im} p(x) \geq 0 .
$$

This inequality will be used in the proof of Claim 2.5 below (see formula (2.21)). It follows from (2.4) that

$$
0=\nabla \cdot\left(K^{2}(x) E\right)=\nabla K^{2}(x) \cdot E+K^{2}(x) \nabla \cdot E .
$$

From (2.9) and (2.10) one gets

$$
-\triangle E-k^{2} E-p(x) E-\nabla(q(x) \cdot E)=0,
$$

where

$$
q(x):=\frac{\nabla K^{2}(x)}{K^{2}(x)}=\frac{\nabla \epsilon^{\prime}(x)}{\epsilon^{\prime}(x)}, \quad q(x)=0 \quad \text { in } D^{\prime} .
$$

From (2.11) and (2.5) one gets

$$
E=E_{0}+\int_{D} g(x, y)\left(p(y) E(y)+\nabla_{y}(q(y) \cdot E(y))\right) d y,
$$

where

$$
g(x, y):=\frac{e^{i k|x-y|}}{4 \pi|x-y|} .
$$

If $q$ vanishes at the boundary of $D$, i.e., $\nabla K^{2}(x)=\nabla p(x)$ vanishes at the boundary of $D$, then, after an integration by parts in the last term of (2.13), and taking into account that

$$
\nabla_{x} g(x, y)=-\nabla_{y} g(x, y)
$$

one gets an equivalent equation:

$$
E=E_{0}+\int_{D} g(x, y) p(y) E(y) d y+\nabla_{x} \int_{D} g(x, y) q(y) \cdot E(y) d y:=E_{0}+T E .
$$

Since we have assumed $K^{2}(x) \neq 0$ and $K^{2}(x) \in C^{2}\left(\mathbb{R}^{3}\right)$, it follows that equation (2.15) is Fredholm equation of the second kind, because the integral operator $T$ in $(2.15)$ is compact in $H^{1}(D)$, where $H^{1}(D)$ is the usual Sobolev space. Indeed, the operator

$$
B f:=\int_{D} g(x, y) f(y) d y
$$

acts from $L^{2}(D)$ into $H^{2}(D)$, and the operator

$$
B_{1} E:=p(y) E+\nabla(q(y) \cdot E)
$$

acts from $H^{1}(D)$ into $L^{2}(D)$. Thus, $T$ acts from $H^{1}(D)$ into $H^{2}(D)$ and is, therefore, compact by the embedding theorem.

Let us summarize in Lemma 2.1 the result we have proved: 
Lemma 2.1. If $K^{2}(x) \in C^{2}\left(\mathbb{R}^{3}\right), K^{2}(x) \neq 0, K^{2}(x)=k^{2}>0$ in the exterior domain $D^{\prime}:=\mathbb{R}^{3} \backslash D$, then the operator $T$ in (2.15) is compact in $H^{1}(D)$, so that equation (2.13) is of Fredholm type in $H^{1}(D)$.

Let us now formulate the second auxiliary result in Lemma 2.2. After its formulation the ideas of the proof are briefly described, The proof consists of proving three claims, that are formulated in the course of the proof.

Lemma 2.2. Equation (2.15) is uniquely solvable in $H^{1}(D)$.

Proof. Let us prove Lemma 2.2. It is sufficient to prove that the homogeneous version of equation (2.15) has only the trivial solution. If $E$ solves the homogeneous equation (2.15), then $E$ solves equation (2.4) and satisfies the radiation condition (2.7). The only solution to (2.4) satisfying (2.7) is the trivial solution $E=0$.

Let us give details and prove the above claims.

Claim 2.3. A solution to (2.15) satisfies equation (2.4), and, consequently, (2.10), that is $\nabla \cdot\left(K^{2}(x) E\right)=0$.

Thus, Claim 2.3 states that (2.15) is equivalent to the original equation (2.4), which is not at all obvious.

Claim 2.4. The only solution to (2.4), (2.7) is $E=0$.

Proof. of Claim 2.3 If $E$ solves (2.15) and is of the form (2.5), then

$$
\left(-\triangle-k^{2}\right) E=p(x) E+\nabla(q(x) \cdot E) .
$$

Rewrite this equation as

$$
\nabla \times \nabla \times E-\nabla(\nabla \cdot E)-\nabla(q(x) \cdot E)=K^{2}(x) E,
$$

or

$$
\nabla \times \nabla \times E-\nabla\left(\frac{K^{2}(x) \nabla \cdot E+\nabla K^{2}(x) \cdot E}{K^{2}(x)}\right)=K^{2}(x) E
$$

Denote

$$
\frac{1}{K^{2}(x)} \nabla \cdot\left(K^{2}(x) E\right):=\psi(x) .
$$

Taking divergence of equation (2.16) one gets

$$
-\triangle \psi-K^{2}(x) \psi=0 \quad \text { in } \quad \mathbb{R}^{3} .
$$

The function $\psi$ satisfies the radiation condition (2.7).

Claim 2.5. The only solution to (2.17), which satisfies condition $(2.7)$, is $\psi=0$.

We prove Claim 2.5 below. Assuming that this claim is proved, we infer that $\psi=0$, so

$$
\nabla \cdot\left(K^{2}(x) E\right)=0
$$

and equation (2.4) holds. Claim 2.3 is proved.

Let us prove Claim 2.5. Equation (2.17) can be written as:

$$
-\triangle \psi-k^{2} \psi-p(x) \psi=0 \quad \text { in } \quad \mathbb{R}^{3},
$$

where $p(x)$ is defined in equation $(2.9)$, and $p(x)=0$ in $D^{\prime}$. It is known that if $k^{2}>0, p(x) \in L^{2}(D)$, $\operatorname{Im} p(x) \geq 0, D$ is a bounded domain, and $\psi$ satisfies the radiation condition (2.7) and equation (2.18), 
then $\psi=0$ (see, e.g., [7]). For convenience of the reader, we sketch the proof. From (2.18) and its complex conjugate one derives the relation

$$
\psi \triangle \bar{\psi}-\bar{\psi} \triangle \psi-2 i \operatorname{Im} p(x)|\psi|^{2}=0 .
$$

Integrate (2.19) over a ball $B_{R}$ centered at the origin of radius $R$, and use the Green's formula to get

$$
\int_{|x|=R}\left(\psi \frac{\partial \bar{\psi}}{\partial r}-\bar{\psi} \frac{\partial \psi}{\partial r}\right) d s-2 i \int_{D} \operatorname{Im} p(x)|\psi|^{2} d x=0 .
$$

By the radiation condition (2.7) for $\psi$ one can rewrite (2.20) as

$$
-2 i k \lim _{R \rightarrow \infty} \int_{|x|=R}|\psi|^{2} d s-2 i \int_{D} \operatorname{Im} p(x)|\psi|^{2} d x=0 .
$$

Since $\operatorname{Im} p(x) \geq 0$ by our assumption, and $k>0$, it follows that

$$
\lim _{R \rightarrow \infty} \int_{|x|=R}|\psi|^{2} d x=0
$$

Relation (2.22) and the equation

$$
\left(\triangle+k^{2}\right) \psi=0 \quad|x|>R_{0},
$$

where $B_{R_{0}} \supset D$, imply $\psi=0$ for $|x|>R_{0}$. (See $[5, \mathrm{p} .25]$ ). By the unique continuation principle for the solutions of the homogeneous Schrödinger equation (2.18), it folows that $\psi=0$. Claim 2.5 is proved.

Let us prove Claim 2.4. This will complete the proof of Lemma 2.2.

If $E$ solves (2.4) and satisfies (2.7), the the pair $\{E, H\}$, where $H$ is defined by the second formula in $(2.2)$, solves the homogeneous Maxwell's system (2.1), satisfies the radiation condition, and $\{E, H\}$ is in $H_{l o c}^{2}\left(\mathbb{R}^{3}\right)$. It is known (see, e.g., [3]) that this implies $E=H=0$. Lemma 2.2 is proved.

From formula (2.15) assuming that the origin is inside $D$, one gets:

$$
E=E_{0}+\frac{e^{i k|x|}}{|x|} \frac{1}{4 \pi} \int_{D} e^{-i k \beta \cdot y}(p(y) E(y)+\nabla(q(y) \cdot E)) d y\left[1+O\left(\frac{1}{|x|}\right)\right]
$$

as $|x| \rightarrow \infty, \frac{x}{|x|}:=\beta$.

An alternative representation of the scattered field is given in Section 3 in formula (3.2).

\section{Inverse scattering for the Maxwell's equations}

We assume that the inhomogeneity is described by the permittivity

$$
\epsilon^{\prime}(x)=\epsilon(x)+i \frac{\sigma(x)}{\omega},
$$

$\epsilon(x)=\epsilon_{0}$ in $D^{\prime}:=\mathbb{R}^{3} \backslash D, \sigma(x)=0$ in $D^{\prime}$, and $D$ is an arbitrary large finite domain, $D \subset B_{R}$, where $B_{R}$ is the ball of radius $R$ centered at the origin. The origin is inside $D$. The inverse scattering problem consists of finding $\epsilon^{\prime}(x)$ from the knowledge of the scattered field measured at large distances from $D$. This scattered field is defined by the scattering amplitude. Let us use equation (2.15) for finding an unknown $\epsilon^{\prime}(x)$ given the scattering amplitude for all $\alpha, \beta$ and a fixed $k>0$. Let us define the scattering amplitude $A(\beta, \alpha, k)$ by the formula

$$
E-E_{0}=\frac{e^{i k r}}{r} A(\beta, \alpha, k)+o\left(\frac{1}{r}\right), \quad r:=|x| \rightarrow \infty, \quad \beta:=\frac{x}{r} .
$$


From equations (2.15) and (3.1) one gets:

$$
A(\beta, \alpha, k)=\frac{1}{4 \pi} \int_{D} e^{-i k \beta \cdot y} p(y) E(y) d y+\frac{i k \beta}{4 \pi} \int_{D} e^{-i k \beta \cdot y} q(y) \cdot E(y) d y .
$$

Denote by $[E, H]=E \times H$ the cross product of two vectors $E$ and $H$, and by $(E, H)=E \cdot H$ their dot product. Then equation (3.2) implies

$$
[\beta, A(\beta, \alpha, k)]=\frac{1}{4 \pi} \int_{D} e^{-i k \beta \cdot y} p(y)[\beta, E(y)] d y .
$$

If one assumes that $|p(y)| \ll k^{2}$, and $E_{0}=\mathcal{E} e^{i k \alpha \cdot x}$, then one may replace $E(y)$ in equation (3.3) by $\mathcal{E} e^{i k \alpha \cdot x}$, and obtain in this approximation, which is similar to the Born approximation in quantum mechanics, the following relation:

$$
4 \pi[\beta, A(\beta, \alpha, k)]=[\beta, \mathcal{E}] \int_{D} e^{i k(\alpha-\beta) \cdot y} p(y) d y .
$$

A novel and practically attractive feature of equation (3.4) consists of a possibility to reduce the inverse problem of finding an unknown permittivity in a Maxwell's system of vector equations to solving a scalar equation (3.7) below. Let us assume without loss of generality that $|\mathcal{E}|=1$. Then $|[\beta, \mathcal{E}]|=\sin \theta$, where $\theta \in[0, \pi]$ is the angle between vectors $\beta$ and $\mathcal{E}$,

$$
([\beta, \mathcal{E}],[\beta, \mathcal{E}])=\sin ^{2} \theta
$$

and (3.4) implies

$$
4 \pi([\beta, A(\beta, \alpha, k)],[\beta, \mathcal{E}]) \sin ^{-2} \theta:=f=\int_{D} e^{i k(\alpha-\beta) \cdot y} p(y) d y .
$$

The right-hand side of (3.5) does not depend on $\theta$. Thus, its left-hand side, $f$, remains bounded for $\theta \in[0, \pi]$. The function

$$
f=f(\beta, \alpha, k)=f(k(\alpha-\beta))
$$

is known since $A(\beta, \alpha, k), \beta, \theta$, and $\mathcal{E}$ are known. Therefore, the inverse scattering problem of finding $\epsilon^{\prime}(x)$ from $A(\beta, \alpha, k)$ is reduced to finding $p=p(y)$ from the data $f(\beta, \alpha, k)$. If $p$ is found, then $\epsilon^{\prime}$ can be found, because

$$
\epsilon^{\prime}(x)=\frac{k^{2}+p(x)}{\omega^{2} \mu},
$$

where $\mu=\mu_{0}$ in $\mathbb{R}^{3}, k^{2}=\omega^{2} \epsilon_{0} \mu_{0}$, and $k^{2}, \omega$ and $\mu_{0}$ are assumed known. Thus, the inverse problem is reduced to solving the scalar equation

$$
\int_{D} e^{i k(\alpha-\beta) \cdot y} p(y) d y=f(\beta, \alpha, k),
$$

where $k=\omega \sqrt{\epsilon_{0} \mu_{0}}>0$ is fixed, $\alpha, \beta \in S^{2}$ run independently of each other through the unit sphere $S^{2}$, and the function $f=f(\beta, \alpha, k)$ is known on $S^{2} \times S^{2}$. let us summarize the result we have proved in Theorem 3.1.

Theorem 3.1. If the scattering amplitude $A(\beta, \alpha, k)$ is known for a fixed $k>0$ and all $\alpha, \beta \in S^{2}$, then one calculates the left-hand side of equation (3.5) thus finding the scalar function $f(\beta, \alpha, k)$, and then calculates the function $p(y)$ by solving equation (3.7).

Let us now give a method for solving equation (3.7). We assume that the data $f$ is given with some error: the exact $f$ is unknown and the "noisy" data $f_{\delta}$ are given such that $\left\|f-f_{\delta}\right\|<\delta$, where $\delta>0$ is a small number. The problem is to find $p=p(y)$ from equation (3.7), given $f_{\delta}$. Such a problem has been 
studied in [5]. We formulate the results from [5] without proof. The reader is referred to the proofs to [5], pp.259-274. The inversion formula from [5], p. 268, is:

$$
p_{N}(x)=\int_{S^{2}} \int_{S^{2}} f\left(k\left(s^{\prime}-s\right)\right) h_{N}\left(k\left(s^{\prime}-s\right)\right) e^{i k\left(s^{\prime}-s\right) \cdot x} d s d s^{\prime},
$$

where $N$ is a positive integer, $s^{\prime}=\alpha, s=\beta, d s$ and $d s^{\prime}$ are surface elements of $S^{2}$,

$$
h_{N}(z):=|z| a_{N}(z) k^{2}\left(32 \pi^{4}\right)^{-1},
$$

where

$$
a_{N}(z):=\int_{\mathbb{R}^{3}} \delta_{N}(x) e^{-i z \cdot x} d x
$$

and

$$
\delta_{N}(x):=\left(1-\frac{|x|^{2}}{4 R^{2}}\right)^{N}\left(\frac{N}{4 \pi R^{2}}\right)^{3 / 2}\left(\frac{\sin b-b \cos b}{b^{3} / 3}\right)^{2 N+3}, \quad b=\frac{2 k|x|}{2 N+3},
$$

where $R$ is the radius of the ball $B_{R}$ containing $D$. It is proved in [5], p.268, that

$$
\left\|p_{N}(x)-p(x)\right\|_{L^{2}\left(B_{R}\right)} \rightarrow 0 \quad \text { as } \quad N \rightarrow \infty .
$$

Suppose now that instead of the exact data $f$ the noisy data $f_{\delta}$ are given, $\left\|f-f_{\delta}\right\|_{L^{2}\left(S^{2} \times S^{2}\right)} \leq \delta$. Then we compute the approximate solution $p_{N \delta}(x)$ by formula (3.4) with $f_{\delta}\left(k\left(s^{\prime}-s\right)\right)$ in place of $f\left(k\left(s^{\prime}-s\right)\right)$, and choose $N=N(\delta)$ according to the recipe on p.269 in [5]. Then $N(\delta) \rightarrow \infty$ as $\delta \rightarrow 0$. With this choice of $N(\delta)$ one proves, as in [5], p. 269, that

$$
\left\|p_{N(\delta)}(x)-p(x)\right\|_{L^{2}\left(B_{R}\right)} \rightarrow 0 \quad \text { as } \quad \delta \rightarrow 0 .
$$

These results were applied by the author also to inversion of incomplete tomographic data (see [11] and [12], pp. 259-264). Let us summarize the result in Theorem 3.2.

Theorem 3.2. A stable solution to equation (3.7) is given by formulas (3.8)-(3.13).

\section{References}

[1] L. Landau, E. Lifschitz, L. Pitaevskii. Electrodynamics of continuous medium. Pergamon Press, Oxford, 1984.

[2] L. Tsang, J. Kong, K.Ding. Scattering of electromagnetic waves: Theories and applications. Wiley, New York, 2000.

[3] C. Müller. Grundprobleme der mathematischen Theorie electromagnetischer Schwingungen. Springer-Verlag, Berlin, 1957.

[4] A. G. Ramm. Wave scattering by small bodies of arbitrary shapes. World Sci. Publishers, Singapore, 2005.

[5] A. G. Ramm. Scattering by obstacles. D.Reidel, Dordrecht, 1986.

[6] A. G. Ramm. Inverse problems. Springer, New York, 2005.

[7] A. G. Ramm. Many-body wave scattering by small bodies and applications. J. Math. Phys., 48, No. 10, (2007), 103511.

[8] A. G. Ramm. Electromagnetic wave scattering by small bodies. Phys. Lett. A, 372/23, (2008), 4298-4306.

[9] A. G. Ramm. Wave scattering by many small particles embedded in a medium. Phys. Lett. A, 372/17, (2008), 30643070.

[10] A. G. Ramm. Wave scattering by many small bodies and creating materials with a desired refraction coefficient. Afrika Matematika, 22, No. 1, (2011), 33-55.

[11] A. G. Ramm. Inversion of the Radon transform with incomplete data. Math.Methods in the Appl.Sci., 15, No. 3, (1992), 159-166.

[12] A. G. Ramm, A.I. Katsevich. The Radon transform and local tomography. CRC Press, Boca Raton, 1996. 